\title{
Emergency Department Sepsis Protocol does not Impact Time to Antibiotics
}

\author{
Meghan E. McGrath $\#$, , Casey M. Rebholz ${ }^{\#, 2}$, Pamela Lada Walker ${ }^{\#, 3}$, Patricia Mitchell ${ }^{\#, 1}$, \\ James Feldman*,\#,1
}

\author{
${ }^{1}$ Boston University School of Medicine, Department of Emergency Medicine, Dowling One South, Boston Medical \\ Center, Boston, MA 02118, USA \\ ${ }^{2}$ Tulane University School of Medicine New Orleans, LA 70112, USA \\ ${ }^{3}$ University of Michigan Hospital, Department of Pharmacy, Ann Arbor, MI, USA
}

\begin{abstract}
Background: Early goal directed therapy (EGDT) can reduce mortality in an Intensive Care Unit (ICU) population with severe sepsis. It is not clear whether EGDT can be effectively implemented in an Emergency Department (ED) with existing resources to achieve the same goals.

Objectives: To assess the impact of EGDT in our ED with existing resources on time to antibiotics (ABX) and patient outcomes.

Methods: We performed a before and after study of the effects of the EGDT protocol on patients over age 21 with severe sepsis admitted to the ICU. Time to ABX was the primary outcome, mortality and other care processes were secondary. Descriptive statistics, Fisher's exact chi-square, and Wilcoxon rank sum tests compared time periods. Multivariate analyses with logistic and Cox proportional hazard regression models were performed.

Results: 192 cases: 91 before and 101 after protocol. Groups were similar in demographics, co-morbidities, severity scores, and overall mortality of $17.7 \%$. Patients with higher Mortality in ED scores (MEDS) received ABX sooner than patients with lower MEDS after adjusting for time period. There was no difference in time to ABX between time periods after adjusting for disease severity (Hazard Ratio: 0.88, 95\% CI: 0.65-1.18; OR: 0.88, 95\% CI: 0.49-1.57).

Conclusion: Our EGDT protocol did not change management, time to ABX, or mortality of septic patients admitted to the ICU, when relying on existing resources. Further study is needed to evaluate barriers to EGDT and feasibility of translating resource intensive protocols to the bedside in routine ED care.
\end{abstract}

Keywords: Sepsis, critical care, drug delivery.

\section{INTRODUCTION}

Severe sepsis is a devastating disease, with an estimated $28 \%$ in-hospital mortality [1]. Sepsis may result in 215,000 annual deaths and cost of $\$ 16.7$ billion nationwide [1]. In 2001, Rivers demonstrated that employing Early Goal Directed Therapy (EGDT), an aggressive strategy aimed to optimize patient hemodynamics, resulted in a $16 \%$ reduction in 28-day mortality when compared with standard care in an Intensive Care Unit (ICU) population [2]. This strategy was time and resource intensive, requiring placement of central venous catheters, invasive blood pressure monitoring, and dedicated physician and nurse teams allocated to the singular care of these patients for six hours. It remained to be seen whether sepsis protocols could be effectively implemented in an Emergency Department (ED) with existing resources to achieve the same goals.

*Address correspondence to this author at the Department of Emergency Medicine Boston Medical Center 818 Harrison Ave. Boston MA 02118, USA; Tel: 617-414-5972; Fax: 617-414-5975; E-mail: jfeldman@bu.edu

"These Authors contributed equally to this work.
The objective of this study was to determine whether implementation of EGDT with existing resources would result in decreased time to antibiotics and improved patient outcomes. Collaborative projects between ED and critical care departments can implement many elements of EGDT [3-5]. A two year implementation of the sepsis bundle increased adherence from zero to $51.2 \%$ and improved mortality from $39.5 \%$ to $20.8 \%$ [6].

We modelled our protocol on previous efforts to improve time to drug therapy for patients with critical illnesses, such as acute myocardial infarction [7-9]. We used time to administration of antibiotics as the primary outcome measure as each hour of delay in antibiotic administration during the first six hours of hypotension in septic patients has been associated with a $7.6 \%$ increase in mortality (range $3.6 \%$ 9.9\%) [10]. Materials and Methods

\section{Study Design}

We performed a before and after study of the effects of EGDT protocol implementation on the treatment of patients 
with severe sepsis treated in the ED and admitted to the ICU. Our project was approved by the hospital Institutional Review Board.

\section{Study Setting and Population}

This study was performed in an urban, level one, academic ED with 128,000 annual patients. We care for a largely low-income population with high rates of homelessness, substance abuse and HIV/AIDS. At any time, there are 6-12 residents, $1-4$ attending physicians and 12-18 nurses in the ED which is similar to other large academic centers. There is a dedicated ED staff pharmacist available for 10-14 hours a day to review orders and assist in rapidly delivering antibiotics. There is a focused team at all times in the ED dedicated to responding to critical care patients made up of two emergency medicine residents, two nurses and 1 attending physician. This team is triggered by the triage nurse and is not specific to septic patients, but is designed to respond rapidly to any critically ill patient, for example, patients requiring emergent intubation, blood products, trauma evaluation, etc. Most patients with sepsis who are stable at the time of triage are not cared for by this specialized team but are cared for in the general ED.

Adult patients (older than 21 years) admitted to the ICU with two or more systemic inflammatory response syndrome criteria were included: temperature less than $36^{\circ} \mathrm{C}\left(96.8^{\circ} \mathrm{F}\right)$ or greater than $38^{\circ} \mathrm{C}\left(100.4^{\circ} \mathrm{F}\right)$, heart rate greater than 90 bpm, respiratory rate greater than 20 or $\mathrm{PaCO}_{2}$ less than 32 $\mathrm{mm} \mathrm{Hg}$, white blood count greater than $12,000 / \mu \mathrm{L}$ or less than $4,000 / \mu \mathrm{L}$ or greater than $10 \%$ bands, systolic blood pressure less than $90 \mathrm{~mm} \mathrm{Hg}$ systolic, and lactate greater than $4 \mathrm{mmol} / \mathrm{L}$. Patients who died in the ED or received intravenous (IV) antibiotics prior to arrival were excluded. Patients designated as comfort care only were excluded.

The EGDT protocol was implemented during January 2006. Septic patients in the ED who were admitted to the ICU between July 1, 2005 and December 31, 2005, constituted the before group and those admitted between February 1, 2006 and July 31, 2006 constituted the after group.

\section{EGDT Protocol}

The EGDT protocol was derived from evidence-based recommendations [11]. Our sepsis bundle was created in collaboration with members of our infectious disease, pharmacy, ICU and ED. It provided guidelines for antibiotic choices for presumed and empiric sources, encouraged early intubation with low tidal volumes, aggressive fluid resuscitation and reviewed indications for vasopressors and transfusion.

All laboratory, imaging and medications require computer orders in our institution. We created a computerized order set designed to help the clinician order appropriate laboratory, imaging and medications in accordance with the sepsis guidelines. We also created web-based resources and pocket cards for staff. The guidelines, computer order sets and pocket cards were introduced to nursing through inservices and to residents and faculty through three hours of case-based lectures and presentations. (Appendices: 1 Order Set, 2 Adult Antibiotics, 3 ED Pocket Card). The staff re- ceived reminders about the guidelines through email, brief discussions before and after shifts, and during conference time. Due to lack of staff resources, we did not perform ongoing case review or monitoring of adherence to the guidelines.

\section{Study Protocol}

Trained research assistants (RAs) screened charts of all ED patients 22 years of age or older who were admitted to the ICU with sepsis. Medical charts were abstracted onto structured data sheets. Six charts were abstracted three times by the RAs and the investigators and standard data abstraction rules were developed. Each chart was abstracted independently by two RAs and reviewed by the investigators; agreement among the multiple data abstractions was not estimated. It was not possible to blind the data abstractors to the study group, as many data points were time and date dependent.

\section{Measurements}

The main outcome measure was time to administration of antibiotics. We also examined the effect of the protocol on processes of care included in the sepsis bundle [11].

\section{Data Analysis}

Descriptive statistics (median, interquartile range) were used for age, severity scores, and length of stay (LOS). We used Fisher's exact chi-square test for categorical variables and Wilcoxon rank sum test for continuous variables to compare time periods. Multivariate analyses with logistic and Cox proportional hazards regression models were performed. For logistic regression, we dichotomized time to antibiotic administration as less than or equal to 2 hours and greater than 2 hours. For Cox proportional hazards analysis, we used a survival model of time to antibiotic administration with time of arrival in the ED censored at time of departure to the ICU. Variables significant at alpha $=0.20$ were included in multivariate models and statistical significance was assessed at alpha $=0.05$ for the final analyses.

A priori sample size calculations were performed and the study was powered to detect an absolute $30 \%$ increase in antibiotic administration within two hours of arrival $(50 \%$ to $80 \%$ ). A sample size of 160 was considered adequate to examine the primary and secondary outcome measures with a power of $80 \%$ at an alpha level of 0.05 . Data were analyzed using SAS version 9.1 (SAS Institute, Cary, NC)

\section{RESULTS}

Of the 1,607 cases admitted to the ICU during the study period, 229 had a diagnosis of sepsis. Of the 229 charts reviewed, 192 were included: 91 before and 101 after protocol (Fig. 1). Groups were similar in demographics, comorbidities and social history (Table 1).

Mortality did not change after protocol implementation (Table 2); $14.3 \%(\mathrm{n}=13)$ died in the before group vs. $20.8 \%$ $(\mathrm{n}=21)$ in the after group $(\mathrm{p}=0.26)$ with an overall mortality of $17.7 \%$. Both groups were critically ill, with similar hemodynamics and Glasgow Coma Scores (Table 1). The median 


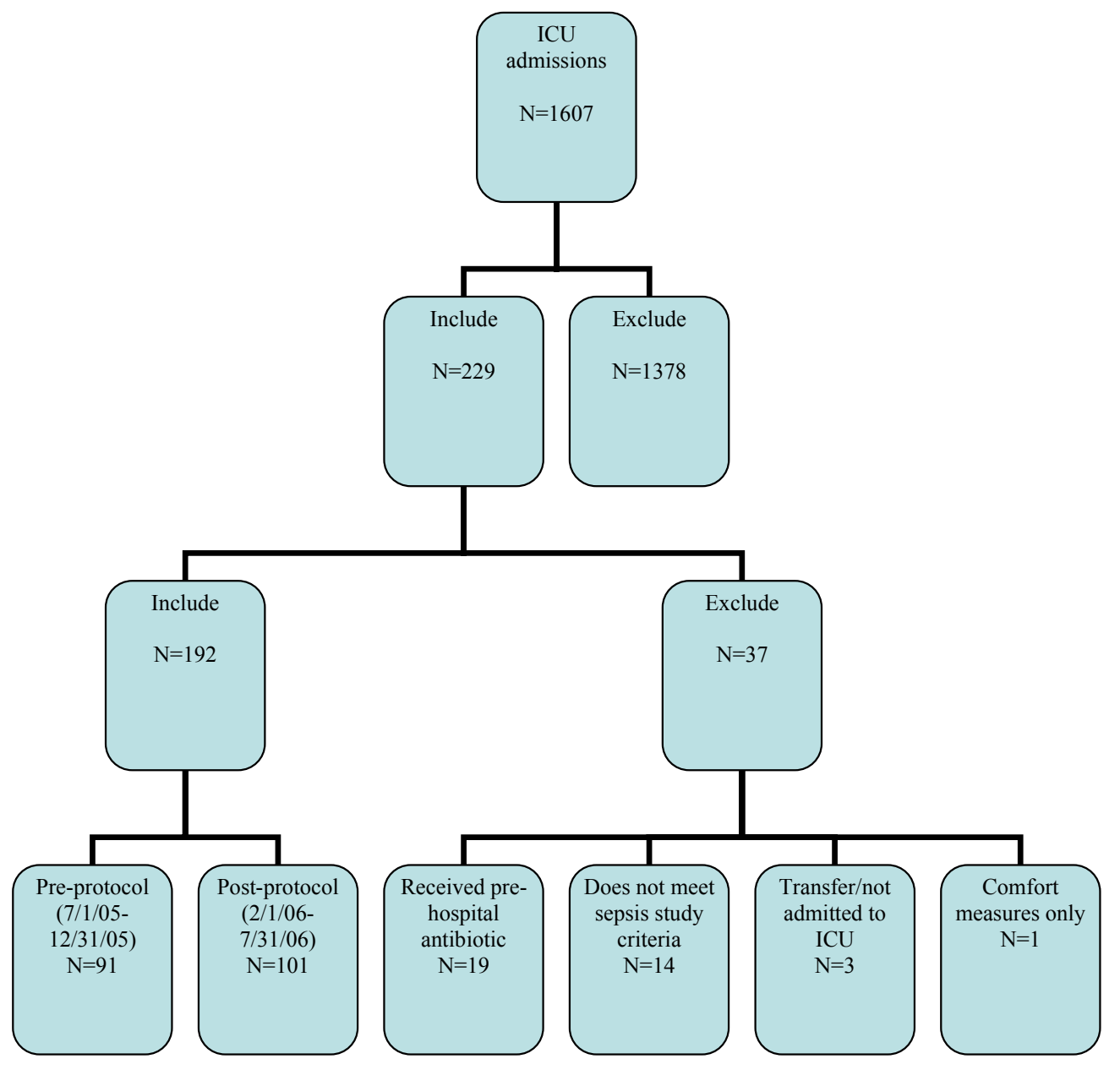

Fig. (1). Study Flow Chart.

Mortality in Emergency Department Sepsis (MEDS) score, as an indicator of illness severity, was 10 (IQR: 6-13). The median modified Rapid Emergency Medicine Score (REMS) was 8 (IQR: 6-10) on a scale of 0 to 23, with higher scores being more predictive of mortality. The median CURB-65 score was 3 (IQR: 1-3.5) on a scale of 0 to 5, with higher numbers being more predictive of mortality. Using CURB$65,49(25.5 \%)$ were in the low mortality group, $42(21.9 \%)$ were in the intermediate mortality group, and $101(52.6 \%)$ in the high mortality group.

Our patients spent a relatively short time in the ED before going to the ICU (Table 2); the median ED length of stay (LOS) was 4.7 hours (IQR: 3.2- 6.5). The median ICU LOS was three days (IQR: 1.2-7.0). ED LOS and ICU LOS were not different before and after protocol implementation.

Management of sepsis did not change in the after group compared to the before group (Table 2). There was no difference in frequency of intubation, central line placement, volume of fluids, use of vasopressors, administration of steroids, or transfusion.

In multivariate analysis, there was no difference in time to antibiotics between the time periods (Table 3). Patients with higher mortality risk (indicated by increasing MEDS score) received antibiotics sooner than patients with lower MEDS scores, after adjusting for time period. There is a $6 \%$ increased odds of receiving antibiotics for each increase in MEDS score, after adjusting for time period (Hazard Ratio: 1.06, 95\% CI: 1.02-1.09), yet there was no statistically significant difference in time to antibiotics between the time periods after adjusting for MEDS score (Hazard Ratio: 0.88, 95\% CI: 0.65-1.18). The after group was as likely as the before group to receive antibiotics within two hours, after adjusting for mortality risk (OR: $0.88,95 \%$ CI: $0.49-1.57)$. In multivariate analysis, insurance, race/ethnicity, or gender did not impact time to antibiotics.

\section{DISCUSSION}

Our EGDT protocol failed to demonstrate a change in management, time to antibiotics, or mortality of septic patients admitted to the ICU. Our study demonstrates the complexity of translating the EGDT guidelines into actual clinical practice. Each of the proposed actions in the sepsis bundle may have specific barriers that should be analyzed and addressed [12-14].

It was reasonable to hypothesize that the multifaceted approach we used could improve time to treatment with antibiotics since previous approaches have been successful in achieving recommended times for delivery of other critical drugs in the ED. However, we observed that only $43.8 \%$ of our patients received antibiotics within the first two hours 
Table 1. Clinical and Demographics Characteristics of Subjects

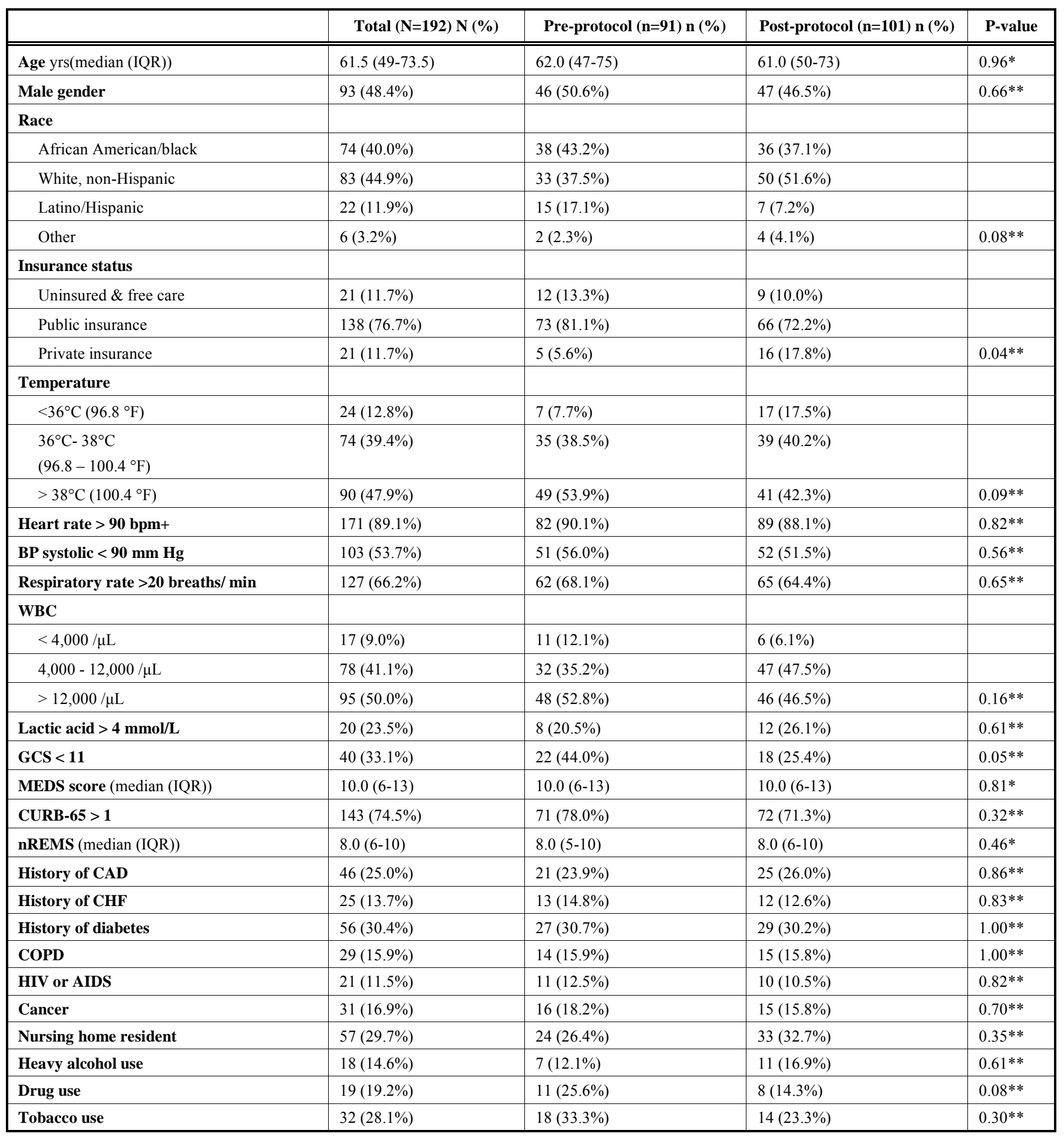

$\mathrm{bpm}+=$ beats per minute.

$\mathrm{WBC}=$ white blood cells.

GCS $=$ Glasgow Coma Scale

MEDS Score $=$ Mortality in Emergency Department Sepsis Score

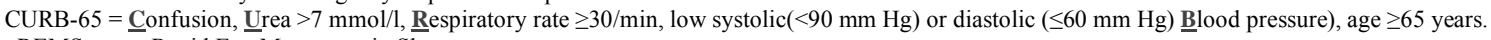

nREMS $=$ non Rapid Eye Movement in Sleep

$\mathrm{CAD}=$ Coronary Artery Disease

$\mathrm{CHF}=$ Congestive Heart Failure

COPD $=$ Chronic Obstructive Pulmonary Disease.

HIV = Human Immunodeficiency Virus.

AIDS $=$ Acquired Immune Deficiency Syndrome.

*P values calculated using Wilcoxon rank sum test.

**P values calculated using chi square or Fisher's exact test. 
Table 2. Comparison of Treatment and Outcomes Pre and Post Implementation of the Sepsis Protocol

\begin{tabular}{|c|c|c|c|c|}
\hline & $\begin{array}{c}\text { Total } \\
(\mathbf{N}=192) \\
\text { N }(\%)\end{array}$ & $\begin{array}{l}\text { Pre-protocol } \\
\qquad \begin{array}{c}(\mathbf{n}=91) \\
\mathbf{n}(\%)\end{array}\end{array}$ & $\begin{array}{c}\text { Post-protocol }(\mathrm{n}=101) \\
\mathrm{n}(\%)\end{array}$ & P-value \\
\hline Urine culture sent & $130(67.7 \%)$ & $65(71.4 \%)$ & $65(64.4 \%)$ & $0.35^{* *}$ \\
\hline Blood culture sent & $169(88.0 \%)$ & $80(87.9 \%)$ & $89(88.1 \%)$ & $1.00 * *$ \\
\hline Central line placed & $50(26.0 \%)$ & $26(28.6 \%)$ & $24(23.8 \%)$ & $0.51 * *$ \\
\hline \multicolumn{5}{|l|}{ Volume of fluids administered } \\
\hline$<2 \mathrm{~L}$ & $48(29.3 \%)$ & $22(29.0 \%)$ & $26(29.6 \%)$ & \\
\hline $2-4 \mathrm{~L}$ & $82(50.0 \%)$ & $38(50.0 \%)$ & $44(50.0 \%)$ & \\
\hline \multicolumn{5}{|l|}{ Antibiotics in ED } \\
\hline Received no antibiotics in ED & $11(5.7 \%)$ & $3(3.3 \%)$ & $8(7.9 \%)$ & \\
\hline Antibiotics ordered but not given & $8(4.2 \%)$ & $3(3.3 \%)$ & $5(5.0 \%)$ & \\
\hline Antibiotics ordered and given & $173(90.1 \%)$ & $85(93.4 \%)$ & $88(87.1 \%)$ & $0.32 * *$ \\
\hline Received $1^{\text {st }}$ dose antibiotic within 2 hours & $84(43.8 \%)$ & $41(45.1 \%)$ & $43(42.6 \%)$ & $0.77 * *$ \\
\hline Received $1^{\text {st }}$ dose antibiotic within 6 hours & $167(87.0 \%)$ & $82(90.1 \%)$ & $85(84.2 \%)$ & $0.28 * *$ \\
\hline Received steroids & $36(18.8 \%)$ & $19(20.9 \%)$ & $17(16.8 \%)$ & $0.58 * *$ \\
\hline ED LOS, hours (median (IQR)) & $4.7(3.2-6.5)$ & $4.8(3.2-6.4)$ & $4.7(3.2-6.7)$ & $0.76^{*}$ \\
\hline
\end{tabular}

ED LOS = Emergency Department Length of Stay.

ICU LOS= Intensive Care Unit Length of Stay.

*P values calculated using Wilcoxon rank sum test.

*** $\mathrm{P}$ values calculated using chi square or Fisher's exact test.

Table 3. Multivariable Analyses of Time to Antibiotic Administration Adjusting for Protocol Time Period and Sepsis Severity

\begin{tabular}{|c|c|c|c|c|}
\hline Model & Factor & Risk Ratio & $95 \% \mathrm{CI}$ & P-value \\
\hline Logistic & Time period & 0.88 & $0.49,1.57$ & 0.5804 \\
\hline \multirow[t]{2}{*}{ Cox PH } & Time period & 0.88 & $0.65,1.18$ & 0.3879 \\
\hline & MEDS score & 1.06 & $1.02,1.09$ & 0.0010 \\
\hline
\end{tabular}

MEDS Score $=$ Mortality in Emergency Department Sepsis Score.

Cox $\mathrm{PH}=$ Cox Proportional Hazard.

only $87 \%$ of patients received antibiotics within the first six hours. Although this proportion compares favorably to other trials, it does not meet our goals. Micek found that standardized order sets resulted in a larger proportion of patients receiving initial antibiotic treatment earlier and reduced inhospital mortality [15]. Our order sets were nearly identical in content but produced dissimilar results. This result highlights the need to examine the effectiveness of strategies to achieve the goals of the sepsis campaign in diverse practice settings.

We found that sicker patients received antibiotics sooner in both the before and after protocol implementation periods. However, some patients received no antibiotics at all in the ED. We found $85 \%$ of antibiotics ordered but not administered in the ED required either special approval from the 
infectious disease department or hand-written orders to the pharmacy. This observation suggests that the implementation of a satellite pharmacy or pre-approved antibiotic orders could reduce treatment delays.

Finally, we relied on existing departmental resources for this project. We did not have dedicated sepsis teams or specialized equipment, as have been employed elsewhere, and we relied on staff already performing their existing duties to implement EGDT [5]. Our implementation phase provided staff education similar in intensity and quality to that described in previous studies [5]. However, we did not provide similar ongoing feedback to clinicians. It is likely that having a dedicated, on-going monitoring system in place would result in better implementation [4,5]. Shapiro has reported that "substantial contribution of time and effort" went into maintaining their protocol, others have reported that dedicated study teams and monitoring systems are needed to ensure compliance $[5,15]$.

Our observed in-hospital mortality rate for sepsis of $17.7 \%$ is lower than mortality rates published elsewhere, even after implementation of EGDT. Specifically, the lowest mortality rate reported after implementing EGDT is $18.2 \%$ and others found rates of $20-30 \%[2,4,5,15]$. We used the same enrolment criteria as other investigators and all of our patients were critically ill and admitted to the ICU. Our median age was 61.5 years, which is younger than the mean age in other studies. Our median MEDS score was 10, which is also lower than the mean MEDS score of 11 reported by Shapiro [5]. It is possible that our sample represented a healthier cohort of sepsis patients which is reflected in our lower mortality rates, or that standard care can achieve mortality rates similar to EGDT.

Following completion of this project, we implemented changes to the ordering system based on these results. Antibiotics formerly requiring specialist approval and hand-

written orders to the pharmacy were moved to the PYXIS medication distribution machine in the ED and nurses were authorized to administer the first dose without awaiting specialist approval. Staff were informed of these results and encouraged to prioritize antibiotic delivery to patients. Residents and nurses were instructed that patients should not be

\section{APPENDIX 1: COMPUTERIZED ORDER SET FOR SEPSIS}

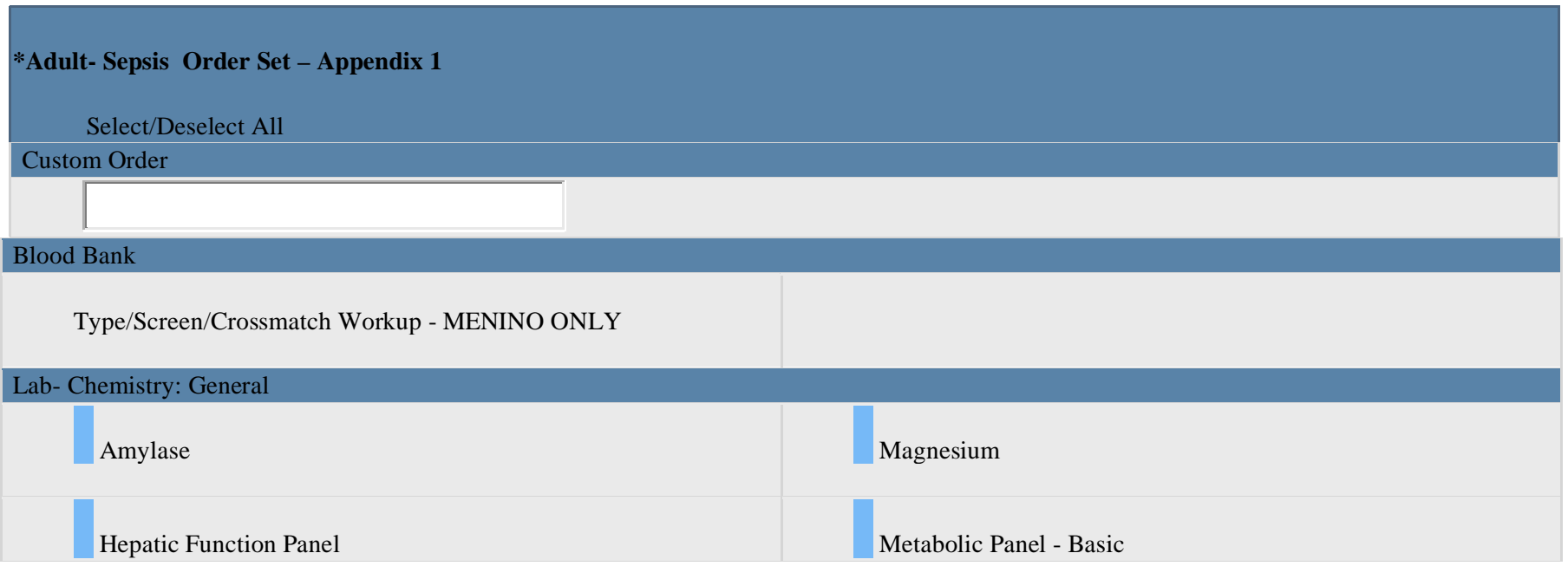

moved to the intensive care unit until antibiotics were ordered and administration had begun.

\section{LIMITATIONS}

Our study was limited by the retrospective design. This design was chosen as we did not feel we could withhold EGDT protocol from a control group. Given that we demonstrated no change in management after our protocol, and a lower than expected mortality rate, it may be appropriate to conduct a prospective randomized trial comparing EGDT with standard care.

Our patients also spent a relatively short time in our ED (less than five hours) as compared to 7.5-13.9 hours reported by others $[2,4,5,15]$. While this short time in the ED may have impacted mortality, it should not have affected our ability to reduce the time to antibiotic administration, the primary outcome measure of this study.

\section{CONCLUSION}

We were unable to demonstrate successful implementation of an EGDT sepsis protocol in our ED utilizing existing resources. Our baseline mortality rate was lower than that reported by others, but no improvement in time to antibiotics or mortality was found after introduction of the protocol. Further study is needed to evaluate barriers to EGDT and feasibility of translating resource intensive research protocols to the bedside.

\section{ACKNOWLEDGEMENTS}

There was no external funding source for this study. Thanks for assistance with data gathering, data entry, and manuscript preparation: H. N. Bullock, J. M. Donovan, K. Shea. This was a poster presentation at the American College of Emergency Physicians Research Forum, October 2008.

\section{CONFLICTS OF INTEREST}

No conflict of interest. 
Lipase

Lab- Chemistry: Hormones - Blood

Cortisol

HCG Pregnancy

Lab- Chemistry: Thyroid Tests

TSH

Lab- Hematology: Coagulation

INR(PT) \& PTT

Lab- Hematology: Routine

CBC with Differential

Lab- Micro: Bacteriology

Culture, Blood

Urine Culture - Routine

Culture, Urine SPTAP/Cysto (Aerobic)

\section{Lab- Urinalysis}

Urinalysis

Rad- X-ray: Chest \& Ribs

Chest Portable X-Ray

.General Nursing Orders

Foley Cath

Obtain IV Access
Saline Lock

\section{.Oxygen Therapy}

Oxygen

.Monitors

Cardiac Monitor

Non-Invasive Blood Pressure Monitor
Pulse-Ox Monitor- 1 time

Pulse-Ox Monitor- continuous

.Point of Care Tests

UHCG Test (Point of Care) 


\section{Lab- Chemistry: Blood Gases}

Arterial Blood Gas w/ Lytes and CR

Lactic Acid (Green Tube on Ice)

IV Pressors and Inotropes

Dobutamine __ug/kg/minute, keep SBP _

Dopamine __ug/kg/minute, keep SBP __
Norepinephrine __ug/minute, keep SBP _

Venous Blood Gas w/ Lytes and CR

Phenylephrine __ug/minute, keep SBP __'

\section{Previous order exists for this service}

\section{APPENDIX 2: COMPUTERIZED ORDER SET FOR ANTIBIOTICS FOR PATIENTS WITH SEPSIS}

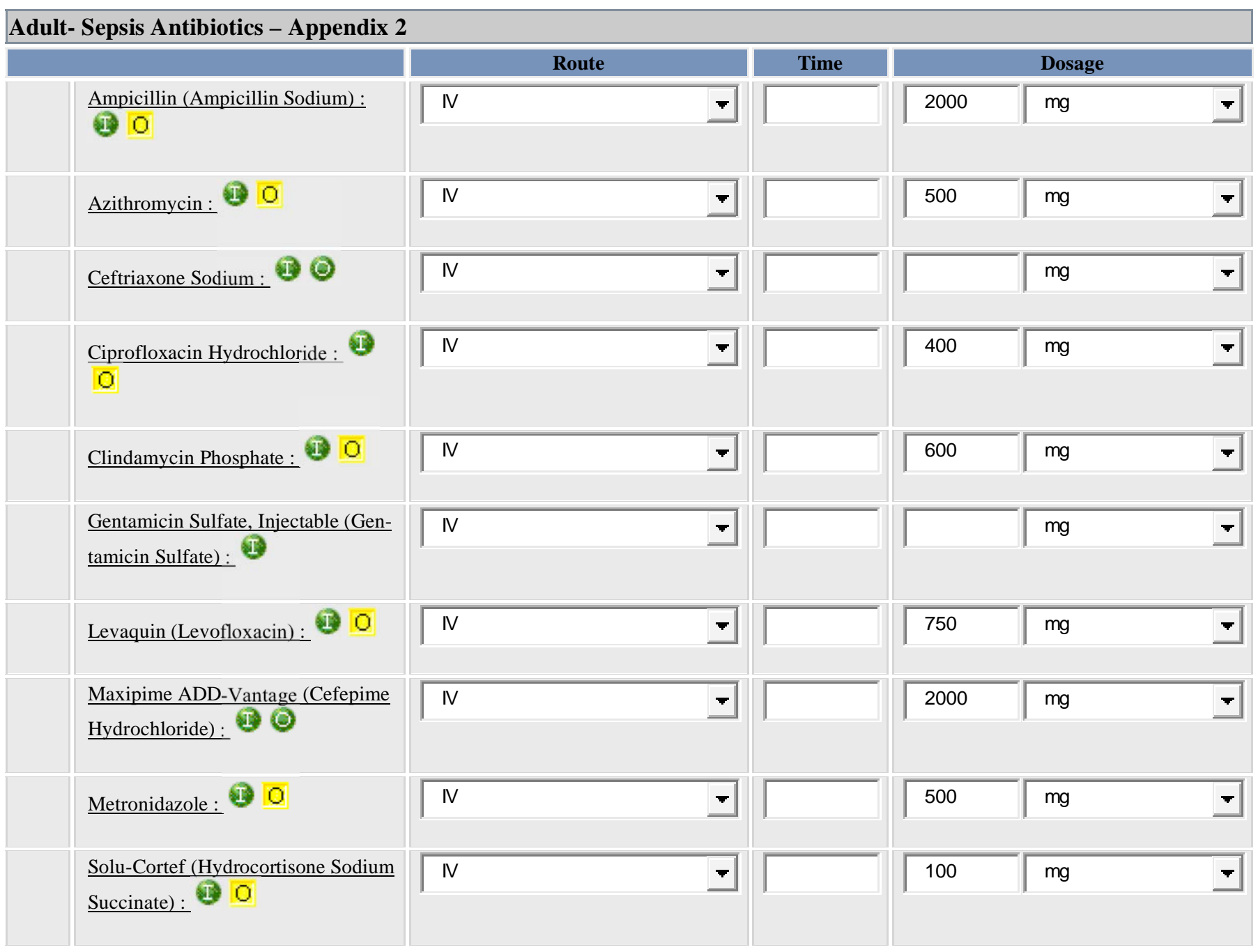




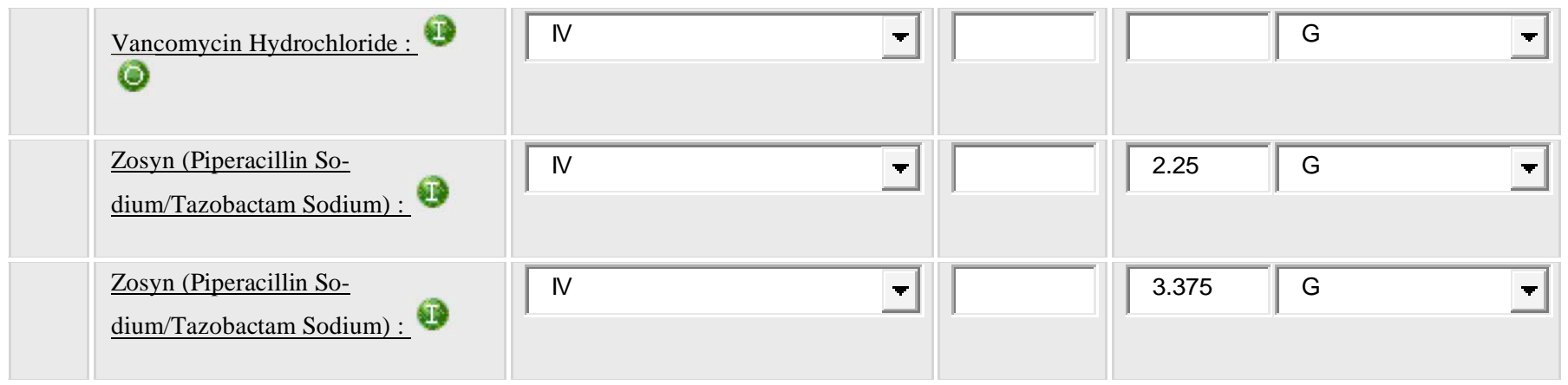

\section{APPENDIX 3: POCKET REFERENCE CARD FOR SEPSIS PROTOCOL}

\section{ED Sepsis Card 2008 - Appendix 3}

BMC ED Adult Sepsis Guidelines 2008

- Untreated sepsis mortality rate $30-50 \%$

- Early aggressive therapy can reduce mortality by $16 \%$

Step 1: Recognize septic shock early

- Temperature $<96.8 \mathrm{~F}(36 \mathrm{C})$ or $>100.4 \mathrm{~F}(38 \mathrm{C})$

- $\quad \mathrm{HR}>90$ beats/min

- $\quad \mathrm{BP}<90$ systolic

- $\quad \mathrm{RR}>20$ or $\mathrm{PaCo} 2<32$

- $\mathrm{WBC}>12,000$ or $<4000$ or $>10 \%$ bands

- $\quad$ Lactate $>4$

- Neuro: lethargy, change in mental status

- Respiratory: hypoxia, SOB, cough

- Renal: low urine output, elevated $\mathrm{Cr}$

- Metabolic: acidosis, elevated lactate, hyperglycemia

Step 2: Consider differential diagnoses of shock

- Alcohol withdrawal, thyroid storm, GI bleed, occult trauma, toxic ingestion,

$$
\text { DKA/AKA, MI }
$$

Step 3: Work-up, move unstable patients to Trauma 1

- $\quad$ Rectal temp

- Portable CXR

- $\quad$ EKG

- Comprehensive Metabolic

- $\mathrm{CBC}$ with differential

- Venous blood gas (ABG if shock or resp compromise)

- Lactate (on VBG or ABG)

- UA, urine culture

- Blood cultures (2 sets)

- Sputum culture (esp. if intubated)

- $\quad$ Type \& cross

- Random cortisol \& TSH

\section{Step 4: Aggressive resuscitation}


- 2 large peripheral IVs or subclavian or IJ central line

- $\quad 2^{\text {nd }}$ choice femoral (incr risk of DVT \& infection)

- $\quad$ Large volume NS (may need 4-10 L) titrate to SBP> 90 and urine output

- Early intubation or non-invasive BiPAP/CPAP

- Low tidal vol $(6 \mathrm{ml} / \mathrm{kg}$ of ideal body wt) in ARDS/ALI

- Elevate head of bed

- Follow urine output

$\mathbf{1}^{\text {st }}$ Line Pressors: if BP refractory to fluids, titrate to MAP $>65$ or systolic $>90$

- Dopamine $5-20 \mathrm{mcg} / \mathrm{kg} / \mathrm{min}$

- $\quad$ Norepinephrine (Levophed) $2-30 \mathrm{mcg} / \mathrm{min}$

\section{$2^{\text {nd }}$ Line Pressors:}

- Vasopressin 0.04 units/min gtt

- $\quad$ Phenylephrine (Neosynephrine) $100-300 \mathrm{mcg} / \mathrm{min}$

- Dobutamine $5-20 \mathrm{mcg} / \mathrm{kg} / \mathrm{min}$

Consider:

- $\quad$ Transfuse $\mathrm{RBCs}$ for $\mathrm{Hb}<8$

- $\quad$ Xigris (needs APACHE-2 $>25$, discuss with MICU)

- Hydrocortisone $100 \mathrm{mg}$ IV (only if known adrenal insuffiency, random cortisol < 15)

- $\quad$ Avoid Na Bicarb unless pH $<7.15$

Guidelines created by: Meg McGrath MD, Tamar Barlam MD, Dana Whitney PharmD, Phil Grgurich PharmD, Art Theodore MD

$$
7 / 20083^{\text {rd }} \text { edition }
$$

\section{ED Adult Sepsis: Early Antibiotics (goal in 1st hour)}

- Clarify PCN/Ceph "allergy" - if not significant, use $1^{\text {st }}$ line antibiotics

- Consult ID (pager 8902 HAC, 8903 ENC) or Antibiotic Management Team (pager 8523)

- $\quad$ ER pharmacy 4-5609 Phil Grgurich, PharmD: pager 8321 Central Pharmacy 47687

\begin{tabular}{|c|c|c|}
\hline Source & $1^{\text {st }}$ line antibiotic & PCN/Ceph allergic \\
\hline $\begin{array}{l}\text { Intra-abdominal Sepsis } \\
\text { Consult surgery early \& consider } \\
\text { CT scan }\end{array}$ & $\begin{array}{l}\text { Zosyn } 3.375 \text { G IV ( } 1^{\text {st }} \text { dose in ED does NOT need ID ap- } \\
\text { proval) }\end{array}$ & $\begin{array}{l}\text { Vancomycin } 15 \mathrm{mg} / \mathrm{kg} \mathrm{IV} \mathrm{+} \mathrm{Flagyl} 500 \mathrm{mg} \mathrm{IV} \mathrm{+} \\
\text { Gentamicin } 6 \mathrm{mg} / \mathrm{kg} \text { IV (high dose, good for } 24 \\
\mathrm{hrs}, 2 \mathrm{mg} / \mathrm{kg} \mathrm{x} 1 \text { dose if renal failure) }\end{array}$ \\
\hline Respiratory & $\begin{array}{l}\text { Ceftriaxone } 1 \mathrm{G} \mathrm{IV}+\text { Azithromycin } 500 \mathrm{mg} \mathrm{IV} \\
\text { If risk for multi-drug resistance: Cefepime } 2 \mathrm{G} \mathrm{IV} \mathrm{+} \mathrm{Vanco-} \\
\text { mycin } 15 \mathrm{mg} / \mathrm{kg} \mathrm{IV} \mathrm{+} \mathrm{Azithromycin} 500 \mathrm{mg} \mathrm{IV}+\text { Gentamicin } \\
6 \mathrm{mg} / \mathrm{kg} \mathrm{IV} \mathrm{(high} \mathrm{dose,} \mathrm{good} \mathrm{for} 24 \mathrm{hrs}, 2 \mathrm{mg} / \mathrm{kg} \mathrm{x} 1 \text { dose if }\end{array}$ & $\begin{array}{l}\text { Levofloxacin } 750 \mathrm{mg} \text { IV } \\
\text { If risk for multi-drug resistance: Levofloxacin } \\
750 \mathrm{mg} \text { IV + Vancomycin } 15 \mathrm{mg} / \mathrm{kg} \text { IV + Gen- } \\
\text { tamicin } 6 \mathrm{mg} / \mathrm{kg} \text { IV (high dose, good for } 24 \mathrm{hrs}, 2\end{array}$ \\
\hline
\end{tabular}




\begin{tabular}{|l|l|l|}
\hline & renal failure) & $\mathrm{mg} / \mathrm{kg}$ x 1 dose if renal failure) \\
\hline Skin/soft tissue & $\begin{array}{l}\text { Toxic shock: Vancomycin } 15 \mathrm{mg} / \mathrm{kg} \text { IV + Clindamycin } 600 \\
\text { mg IV } \\
\text { Necrotizing fasciitis: Vanc + Clinda + Zosyn 3.375 G IV }\end{array}$ & $\begin{array}{l}\text { Toxic shock: Vancomycin 15 mg/kg IV +/- Clin- } \\
\text { damycin } 600 \mathrm{mg} \text { IV } \\
\text { Necrotizing fasciitis: Vanc + Clinda + Gentamicin } \\
2 \mathrm{mg} / \mathrm{kg} \mathrm{IV}\end{array}$ \\
\hline Catheter/IVDU/ endocarditis & Vancomycin $15 \mathrm{mg} / \mathrm{kg} \mathrm{IV} \mathrm{+Gentamicin} 2 \mathrm{mg} / \mathrm{kg} \mathrm{IV}$ & $\begin{array}{l}\text { Vancomycin } 15 \mathrm{mg} / \mathrm{kg} \mathrm{IV} \mathrm{+} \mathrm{Gentamicin} 2 \mathrm{mg} / \mathrm{kg} \\
\text { IV }\end{array}$ \\
\hline CNS & $\begin{array}{l}\text { Ceftriaxone 2 G IV + Vancomycin } 15-20 \mathrm{mg} / \mathrm{kg} \mathrm{IV} \mathrm{+/-} \mathrm{Am-} \\
\text { picillin 2 G IV (if suspect Listeria) }\end{array}$ & Page ID if CNS infxn and true PCN allergy \\
\hline
\end{tabular}

\section{REFERENCES}

[1]

Angus DC, Linde-Zwirble WT, Lidicker J, et al. Epidemiology of severe sepsis in the United States: analysis of incidence, outcome, and associated costs of care. Crit Care Med 2001; 29: 1303-10.

[2] Rivers E, Nguyen B, Havstad S, et al. Early goal-directed therapy in the treatment of severe sepsis and septic shock. N Engl J Med 2001; 345:1368-77.

[3] De Miguel-Yanes JM, Andueza-Lillo JA, Gonzalez-Ramallo VJ, et al. Failure to implement evidence-based clinical guidelines for sepsis at the ED. Am J Emerg Med 2006; 24(5): 553-9.

[4] Trzeciak S, Dellinger RP, Abate NL, et al. Translating research to clinical practice: a 1-year experience with implementing early goaldirected therapy for septic shock in the emergency department. Chest 2006; 129: 225-32.

[5] Shapiro NI, Howell MD, Talmor D, et al. Implementation and outcomes of the Multiple Urgent Sepsis Therapies (MUST) protocol. Crit Care Med 2006; 34: 1025-32.

[6] Nguyen HB, Corbett SW, Steele R, et al. Implementation of a bundle of quality indicators for the early management of severe sepsis and septic shock is associated with decreased mortality. Crit Care Med 2007; 35(4): 1105-12.

[7] Bradley EH, Herrin J, Wang Y, et al. Door-to-drug and door-toballoon times: where can we improve? Time to reperfusion therapy in patients with ST-segment elevation myocardial infarction (STEMI). Am Heart J 2006; 151(6): 1281-7.
[8] Cannon CP, Johnson EB, Cermignani M, et al. Emergency department thrombolysis critical pathway reduces door-to-drug times in acute myocardial infarction. Clin Cardiol 1999; 22(1): 17-20.

[9] McLean S, O'Reilly M, Doyle M, et al. Improving Door-to-Drug time and ST segment resolution in AMI by moving thrombolysis administration to the Emergency Department. Accid Emerg Nurs 2004; 12(1): 2-9.

[10] Kumar A, Roberts D, Wood KE, et al. Duration of hypotension before initiation of effective antimicrobial therapy is the critical determinant of survival in human septic shock. Crit Care Med 2006; 34(6):1589-96.

[11] Dellinger RP, Carlet JM, Masur H, et al. Surviving Sepsis Campaign guidelines for management of severe sepsis and septic shock. Crit Care Med 2004; 32(3): 858-73.

[12] Grimshaw JM, Eccles MP, Walker AE, et al. Changing physicians' behavior: what works and thoughts on getting more things to work. J Contin Educ Health Prof 2002; 22(4): 237-43.

[13] Grimshaw JM, Eccles MP. Is evidence-based implementation of evidence-based care possible? Med J Aust 2004; 180 (6 Suppl): S50-S1.

[14] Grimshaw JM, Thomas RE, MacLennan G, et al. Effectiveness and efficiency of guideline dissemination and implementation strategies. Health Technol Assess 2004; 8(6):iii-iv: 1-72.

[15] Micek ST, Roubinian N, Heuring T, et al. Before-after study of a standardized hospital order set for the management of septic shock. Crit Care Med 2006; 34: 2707-13.

Received: October 04, 2011

Revised: November 17, 2011

Accepted: November 21, 2011

(C) McGrath et al.; Licensee Bentham Open.

This is an open access article licensed under the terms of the Creative Commons Attribution Non-Commercial License (http://creativecommons.org/licenses/ by-nc/3.0/) which permits unrestricted, non-commercial use, distribution and reproduction in any medium, provided the work is properly cited. 